\title{
Evaluating Three Scrutability and Three Privacy User Privileges for a Scrutable User Modelling Infrastructure
}

\author{
Demetris Kyriacou, Hugh C Davis, and Thanassis Tiropanis \\ Learning Societies Lab \\ School of Electronics and Computer Science, University of Southampton \\ Highfield, Southampton, SO17 1BJ, United Kingdom \\ $\{$ dke06r, hcd, tt2 $\} @$ ecs.soton.ac.uk
}

\begin{abstract}
This paper describes the evaluation of a Scrutable User Modelling Infrastructure. SUMI is intended to form a service to allow users to share their user models from social e-networking and e-commerce providers to educational systems. The model is scrutable, meaning users can inspect and correct the data that is held about them, and implements privacy policies so that users can control how their models are accessed by other users. This evaluation was conducted with 107 users, which were exposed to a prototype service, for determining whether the proposed scrutability and privacy privileges were acceptable to the users, whether the users were able to achieve the desired outcome, and whether they understood the consequences of their interactions with the system. The conclusions show that the users expressed their general approval of the proposed privileges while making useful suggestions regarding improvements to the presentation and interface to the system.
\end{abstract}

\section{Introduction}

Our research has revolved around gathering the requirements for adopting a Scrutable User Modelling Infrastructure (SUMI) for the e-commerce and social enetworking domains, in order to enable exchanging of user models among these domains and educational personalization systems, in an attempt to enrich the various sets of user information which are being used for adaptation purposes. We have focused on three key User Modelling 'ingredients' - interoperability, scrutability and privacy. In this paper we present our work on scrutability and user privacy while attempting to answer the following research question: To what extend is it possible for such an infrastructure to allow users to scrutinize the modelling process and express their data privacy preferences?

\section{Key User Modelling Components \& Identified Problem}

Lifelong User Modelling: User Modelling (UM) is the 'heart' of educational personalization services such as AHA!, which offers adaptive content through fragment variants and adaptive link presentation [1]. By keeping a model for each 
user, it allows unique adaptation and presentation of the available resources based on these models, thus enabling successful interactions between users and personalization systems. Lifelong UM was introduced in an attempt to model users' daily-lifelong interactions with several services on the World Wide Web (WWW) while offering to the users the ability to scrutinize and control the whole personalization process [2].

Scrutability: The term scrutability in user modelling signifies that every user's model can be inspected and altered by its owner in order to determine what should be modelled about him/her and how that modelling and following personalization process will be conducted. Scrutable solutions allow users to inspect and alter the value of any single inference that is used for drawing conclusions about them [3].

Privacy-Enhanced Personalization: An area that aims at merging together the techniques and goals of UM with privacy considerations and apply the best possible personalization inside the boundaries set by privacy rules. As the research shows, there is no ideal solution while attempting to combine these two crucial elements. Instead, numerous small enhancements can be implemented, depending on the user and application domains in each case, in order to achieve the best possible solution[4].

Identified Problem: The area of UM is undoubtedly progressing. But, while we find UM in a state of transition, is still been applied single-dimensionally: Most adaptive systems developed, are only using their internal models when offering personalization services to their users. In addition, newly introduced frameworks and architectures, while offering a solution in achieving interoperability across peer systems, do not involve systems beyond the educational domain. Furthermore, User Modelling Servers, a client-server architecture for allowing central information storing and simultaneously data access and retrieval, although are considering and offering scrutability and privacy options to their users, are mostly designed and developed to meet commercial requirements [5]. We are loosing user information, which is flowing on the WWW, because we are not thinking multi-'domain' sionally. We can enrich UM if we find a way to model our every day (life-long) interactions with services from the social e-networking and the e-commerce domains, in order to enrich user information sets which are used in the educational domain for personalization purposes. Recent data portability announcements from two key players in the social e-networking domain $[6,7]$ which revealed these providers' initiatives to pass user data back to their 'owners' have made this multi' domain' sional vision even more feasible.

\section{SUMI User Evaluation}

SUMI's goal is to allow users to gather their various models which they hold with several social e-networking and e-commerce providers, and interact with these models via a SUMI service, using a set of offered scrutability and privacy privileges. Special consideration has been given to collecting the requirements for employing such an infrastructure in an attempt to enrich the current picture in UM [8].

Achieving Interoperability: In this paper we have focused on presenting our work on scrutability and user privacy, thus we will not expand on our solution for achieving interoperability across the social e-networking and e-commerce domains, which is 
undoubtedly as important as considering scrutability and privacy user privileges. We will just briefly mention that this has been achieved using a 4-category models' architecture which resulted after comparative evaluations of representatives of both domains, a SUMI ontology [9], which uses dictionary concepts to define meaning and a RESTful approach as communication protocol. Providers of user models can describe their data model inside SUMI, in order to allow users to import them in SUMI and for educational services to express interest by subscribing to them.

Proposed Scrutability \& Privacy Privileges: In this paper, emphasis is given in our work on scrutability and user privacy which the first user evaluation is based on. More specifically we have proposed:

Three SCRUTABILITY user privileges for SUMI which were exposed to users in the form of three tasks: Task 1: Users were asked to add at least one social e-networking and e-commerce model to their SUMI collection. Users were exposed to the 4category SUMI model's architecture. Task 2: In this task users were asked to import the content of their previously added models in two ways, dynamically-meaning realtime HTTP GET requests and retrieval of real-time data from the provider of the user model using the provider's API, and statically-meaning the cache copy that was taken when the last dynamic import request was generated by the user, and will be retrieved from the SUMI database using SQL queries. Network failures or busy network traffics, are some reasons that users could take advantage of the static import option. Task 6: During this task users were asked to respond to a request and export the content of one of their models to a group formation system, while inspecting and approving, during the export process, all transaction details.

Three PRIVACY user privileges for SUMI which were exposed to users in the form of three tasks: Task 3: During this task, users had to set the privacy status of all 4 categories of at least one of their models using the proposed 3 privacy settings: public-others can see that the model exists and anyone can view its content, privateothers can see that the model exists but they have to place a request to the model's owner for viewing the model's content, and hidden-others can not see that the model exists, therefore the model's content is accessed only by the model's owner. Task 4: Users had to respond to another user's viewing request and allow the requester to view the content of the requested model. Task 5: Users were asked to visit another user's SUMI collection of models and place a request on one private category of that user's models.

Evaluation Objectives: In order to properly evaluate our work, we prepared a prototype SUMI service which has been designed for evaluation and demonstration purposes [10]. The objective of the evaluation was to evaluate if the proposed scrutability and privacy user privileges are appropriate to be offered in SUMI and accepted by SUMI users. For the purposes of this evaluation we have defined the terms "appropriate" and "accepted" as follows: Appropriate-Adequate to satisfy a user need; fit for purpose. Accepted-Generally approved or recognized. In order to evaluate if the proposed privileges were appropriate to be offered in SUMI we took into account the combination of: a) users' competence on completing each presented task which exposed the proposed privileges (we compared the actual outcome after the completion of the tasks with the users' answers to the evaluation questions - have they done it VS. do they think they have done it) and b) users' understanding of the consequences of their decisions while interacting with each task- thus interacting with 
the proposed privileges. In addition, in order to evaluate if the proposed privileges were accepted by SUMI users we tested users' acceptance of the proposed privileges by asking them directly what they think about them and if they would like SUMI to offer them to its users.

Participants \& Evaluation Structure: Our target audience was any undergraduate and postgraduate student of any study discipline and age range. Participants were approached via online forums and social networking groups. A sample size of 107 participants was achieved during a 30-day evaluation process. The evaluation consisted of three parts: First, a pre-questionnaire allowed us to classify how much users knew about their scrutability and privacy options while interacting with various social e-networking and e-commerce providers on the WWW. Second, six tasks exposed to the participants all proposed scrutability and privacy privileges. Users were asked to complete all 6 tasks, while answering some questions during their interaction with each one of them. Furthermore, after the completion of all 6 tasks, users were asked to complete a series of 3-questions-per-task which helped us identify the degree of competence, understanding of consequences, and acceptance for each proposed privilege. Third, a post-questionnaire revealed valuable conclusions regarding: how much users valued scrutability and privacy after the completion of the evaluation, users' proposals for any new scrutability and privacy privileges, what users think about SUMI as a service, and finally what users think about the fact that SUMI is keeping a copy of their data while interacting with it.

\section{Evaluation Results}

Pre-Questionnaire: The pre-questionnaire exposed some useful lessons regarding the users' familiarity with the terms scrutability and user privacy. Results have shown that $89 \%$ of students do not know what the term scrutability means, although $38 \%$ can easily identify some scrutability privileges once they have been explained to them. Furthermore, $80 \%$ of users have found the idea of having scrutability privileges available when interacting with various providers to be a very good idea, which shows the recognition of how important scrutability is to users, once explained to them. User privacy is a term more familiar to users than scrutability. It is something $64 \%$ of users understand and recognize when interacting with several providers, although $32 \%$ of participants choose not to take advantage of it. But, at the suggestion of not having any privacy privileges available, $91 \%$ of users expressed their concerns.

Scrutability \& User Privacy Privileges - Comparison of Results: Table 1 summarizes the results and conclusions regarding which privileges have been successful in which category - competence, consequence and acceptance. The $\mathbf{X}$ symbol means "not satisfactory", where $\checkmark$ marks "success".

Table 1. Results (\% of successful responses) \& conclusions for the proposed user privileges

\begin{tabular}{lcclll}
\hline Task1: & Task2: & Task6: & Task3: & Task4: & Task5: \\
Adding & Importing & Exporting & Setting & Responding & Placing \\
models & content & content & privacy & to viewing & viewing \\
& & & status & request & request \\
\hline
\end{tabular}




\begin{tabular}{lllllll}
\hline Competence & $100 / 100(\checkmark)$ & $79 / 60(\mathbf{X})$ & $91 / 100(\checkmark)$ & $76 / 68(\mathbf{X})$ & $88 / 74(\mathbf{X})$ & $84 / 100(\checkmark)$ \\
Consequence & $77(\mathbf{X})$ & $67(\mathbf{X})$ & $60(\mathbf{X})$ & $100(\checkmark)$ & $100(\checkmark)$ & $79(\checkmark)$ \\
Acceptance & $89(\checkmark)$ & $100(\checkmark)$ & $94(\checkmark)$ & $100(\checkmark)$ & $99(\checkmark)$ & $83(\checkmark)$ \\
During & 73 & 78,70 & 72 & 71 & N/A & 100
\end{tabular}

Competence's results show: $\%$ of users who answered positively to the question "do you think you have managed to complete the task?" /\% of actual successful outcome

Competence: It was clear from the results of the competence questions combined with the results of the during-task questions, that scrutability tasks 1 and 6 and privacy task 5 can be completed successfully by our target audience. It is also clear that scrutability task 2 and privacy tasks 3 and 4, although the responses were satisfactory, users' confidence \% did not meet the actual outcome \% and this begs the question of how much our presentation format affected these results.

Consequence: A different picture appears when we compare the successful responses on the consequence questions. All 3 tasks of privacy have returned acceptable percentages, where the results for the 3 scrutability tasks show that users require further education regarding those privileges. This can be explained from the fact that all today's social e-networking providers, such as Facebook and MySpace offer similar privacy privileges to their users. On the other hand, scrutability privileges are not so popular among providers thus users do not have the same level of familiarity and knowledge. In addition, some technical terminology which was presented to the users may be found too abstract and difficult to understand.

Acceptance: The most important conclusions were revealed after our comparative evaluation of the results on the acceptance questions. All 6 privileges were accepted by an average of $94 \%$ of our targeted audience. The confidence interval for a confidence level of $95 \%$ is 4.44 . This is a very important conclusion which proves our acceptance hypothesis.

Post-Questionnaire: Finally the post-questionnaire revealed participants' highly positive attitude about the SUMI service which they were exposed to. $92 \%$ of users approved our work which was reflected in the evaluation, although $39 \%$ of them did not fully agree with the feature of SUMI keeping a copy of their information inside its databases. Two important results of this evaluation can be identified in the participants' responses on the last two questions. $85 \%$ and $79 \%$ chose the best answer available when asked, after the completion of the evaluation, how much they valued scrutability and user privacy respectively. If these percentages were to be compared with the responses in the pre-questionnaire, and specifically with the responses in the questions regarding how much users were familiar with the two terms before the evaluation, we observe a significant raise of percentages in both occasions; familiar with scrutability options in pre-questionnaire: $10 \%$, appreciation percentage in postquestionnaire: $85 \%$, familiar with privacy options in pre-questionnaire: $64 \%$, appreciation percentage in post-questionnaire: $79 \%$.

\section{Main Conclusion \& Future Work}

As our results have shown, although the proposed infrastructure meets the requirements to be considered as the solution to the identified problem, technical 
complexity and inadequate presentation formats caused the low percentages of successful responses in some tasks. This will be the starting point of our upcoming schedule. In addition, many useful suggestions came out from the evaluation:

Scrutability: $22 \%$ of users raised an important issue when they asked us to find a way so that subscribed services would inform users how their data would be used before users deciding if they would go through with the export transaction or not. Moreover, $43 \%$ of users expressed their concerns regarding SUMI keeping a copy of their information when initiating a dynamic import of their content. Some of them asked us to introduce an on/off switch so they could set which imported attributes SUMI will be allowed to keep internally, while others requested the option of deleting attributes' values from SUMI after inspection of their SUMI collection of models.

User Privacy: The main suggestion we received for user privacy from $37 \%$ of the participants was to allow them to create groups of users and assign a common privacy status. In addition, $13 \%$ of participants requested two more privacy settings, "block" and "ignore", which could be added to SUMI's current set of privacy settings. Finally, $31 \%$ of users wanted to be allowed to take back the viewing access they have granted to another user when they responded to the viewing request in task 4.

After we go through this already-set agenda, we will conduct a second SUMI user evaluation in order to properly test any changes we decide to make. Results should expose a significant improvement of the identified weak areas and the percentages of successful responses should be higher in order to confidently claim that we have proven all of our hypotheses.

\section{References}

1. De Bra, P., Calvi, L., AHA: a Generic Adaptive Hypermedia System, In Proc. of the 2nd Workshop on Adaptive Hypertext and Hypermedia, Pittsburgh, 5--12 (1998)

2. Kay, J., Lifelong user models, memory and learning. ResearchChannel.org, Last retrieved on 29/03/2009 at http://www.researchchannel.org/prog/displayevent.aspx?rID=21195\&fID=4752, (2007)

3. Kay, J., Scrutable adaptation: because we can and must. In V. Wade, H. Ashman, and B. Smyth, editors, Proceedings of Adaptive Hypermedia and Adaptive Web-Based Systems, 4th International Conference, AH2006, pages 11-19. Springer, (2006).

4. Kobsa, A., Privacy-Enhanced Personalization. In Communications of the ACM, 50(8), 24--33 (2007)

5. Fink J., Kobsa, A., A Review and Analysis of Commercial User Modeling Servers for Personalization on the World Wide Web. In User Modeling and User-Adapted Interaction - Special Issue on Deployed User Modeling, 10, 209--249 (2000)

6. Facebook Developers News, Announcing Facebook Connect, Last Retrieved on 29/03/2009 at http://developers.facebook.com/news.php?blog=1\&story=108

7. Google Inc, Google Friend Connect, Last Retrieved on 29/03/2009 at http://www.google.com/friendconnect

8. Kyriacou D., A Scrutable User Modelling Infrastructure for enabling life-long User Modelling. In Adaptive Hypermedia and Adaptive Web-Based Systems - Doctoral Consortium, Hannover, Germany, (2008)

9. SUMI Ontology, Last Uploaded on 29/03/2009 at http://mysumi.org/sumiOntology.owl

10. mysumi.org - SUMI prototype service, http://mysumi.org/1stEvaluation.html 